\section{FACTORS INFLUENCING THE SUCCESS RATE OF PATIENT DERIVED XENOGRAFT FORMATION FROM BREAST CANCER SPECIMENS}

Y. Yang ${ }^{1}$, C. Cooper ${ }^{2}$, E. Robbins ${ }^{3}$, A. Swarbrick ${ }^{4}$, K. Harvey ${ }^{4}$, E. $\mathrm{Lim}^{4}$, C. Mak ${ }^{5}$, H. Carmalt ${ }^{5}$, S. Warrier ${ }^{5}$, B. Chan ${ }^{5}$, J. Beith ${ }^{5}$, M. Hui ${ }^{5}$, L. Gluch ${ }^{1}$, S. O'Toole ${ }^{4,6}$, W. Cooper ${ }^{1,3,7}$

${ }^{1}$ Sydney Medical School, University of Sydney, Australia; ${ }^{2}$ Princess Alexandra Hospital, Brisbane, Australia; ${ }^{3}$ Tissue Pathology and Diagnostic Oncology, Royal Prince Alfred Hospital, Sydney, Australia; ${ }^{4}$ Garvan Institute Medical Research/KHCC, Sydney, Australia; ${ }^{5}$ Chris O'Brien Lifehouse, Sydney, Australia; ${ }^{6}$ Australian Clinical Laboratories, Sydney, Australia; and ${ }^{7}$ School of Medicine, University of Western Sydney, Sydney, Australia

Background: Patient derived xenografts (PDX) have been increasingly used as models for cancer research because they more closely resemble the disease than cancer cell lines. However, there is often high cost associated with PDX partly due to its low engraftment rate.

Aims: In this study, we aimed to investigate clinicopathological features of specimens from breast cancer patients that may influence success of xenograft formation in mice.

Methods: Specimens were obtained from patients at the Royal Prince Alfred Hospital and transplanted into immuno-deficient mice. The mice were monitored for one year to determine whether or not engraftments were successful. Clinicopathological features were correlated with engraftment outcomes.

Results: A total of 45 PDX were performed in this study. There were strong negative correlations between engraftment rate and oestrogen receptor and progesterone receptor expression in tumours $(p<0.001)$. Xenograft formation positively correlated with triple negative breast cancer $(p<0.001)$, and high mitotic count of the tumour $(p=0.0025)$. No other significant correlations were found between engraftment rate and other clinicopathological parameters including age, family history of breast or ovarian cancer, grade of tumour, tubule formation, nuclear pleomorphism, lymphatic invasion, HER2 receptor status, Ki-67 proliferative index, or $\mathrm{T}$ and $\mathrm{N}$ staging.

Conclusions: Tumours lacking oestrogen and progesterone receptor expression and with high mitotic counts are more suitable for xenograft formation.

\section{PRIMARY ANGIOSARCOMA OF THE BREAST IN A 29-YEAR OLD FEMALE}

G. Valloso, L. Cale-Subia

Philippine Heart Center, Quezon City, Philippines

Background: Primary angiosarcoma of the breast is a relatively rare entity with less than $0.05 \%$ of all malignant breast tumours. Aims: Provide in-depth study of a 29 -year-old female with primary angiosarcoma of the breast and review related literature.

Methods: Review all the data which include patient's demographic, history, radiographic findings, management, histopathology findings, and outcome.

Results and conclusions: Case of a 29-year-old Filipino female with nine months history of right breast mass. Work-up favoured breast haemangioma versus phyllodes tumour. Patient underwent right modified radical mastectomy with axillary lymph node dissection. The specimen revealed a unilocular space measuring
$17 \times 12 \times 12 \mathrm{~cm}$ and partly-filled with a mixture of clotted blood and spongy to soft black materials which were adherent to the cavity wall. Microsections showed an infiltrating neoplasm of anastomosing blood-filled vascular channels with prominent papillary growth of neoplastic endothelial cells extending $0.5 \mathrm{~cm}$ away from the basal margin. The neoplastic endothelial cells were pleomorphic with large, round to ovoid, and vesicular nuclei, prominent nucleoli, and eosinophilic cytoplasm. Mitosis was frequent ( $>19 / 10 \mathrm{HPF})$. Necrosis was nil. All nine lymph nodes were negative for involvement. The neoplastic cells showed strong reactivity against CD34 and focal reactivity against Factor VIII. Surgical pathology diagnosis was a conventional angiosarcoma, FNCLCC grade 2. Cycles of chemotherapy (paclitaxel, doxorubicin, and ifosfamide) were also started. Seven months post-operation CT-scan reveal liver masses. The disease progressed and she eventually died a year after.

Studies to verify the correlation between histopathologic grade, clinical and radiologic data, and patient outcomes are needed to develop the most reliable treatment options.

Reference

1. Bordoni D, Bolletta E, Falco G, et al. Primary angiosarcoma of the breast. Int J Surg Case Rep 2016; 20 S (Suppl): 12-5.

\section{COLLISION TUMOUR: AMELANOTIC LENTIGO MALIGNA AND ATYPICAL FIBROXANTHOMA/ PLEOMORPHIC DERMAL SARCOMA (AFX/PDS): A CASE REPORT}

\author{
A. Spasich ${ }^{1}$, K. Manoharan ${ }^{1}$, B. Dutta ${ }^{2}$ \\ ${ }^{1}$ Laverty Pathology, Sydney, Australia; and ${ }^{2}$ Kossard \\ Dermatopatholgists, Sydney, Australia
}

Background and aims: Collision tumours in the skin are unusual neoplastic events. They include carcinosarcoma, Merkel cell carcinoma with squamous cell carcinoma, basal cell carcinoma and Bowen's disease and squamo-melanocytic tumour. Collision tumours involving lentigo maligna, malignant melanoma and AFX/PDS are extremely rare.

Aims: Here we present a case of a collision tumour of lentigo maligna and AFX/PDS.

Methods: A 90-year-old male presented with a scaly, erythematous plaque on the vertex of his scalp. The approximate size of the lesion was $15 \times 12 \mathrm{~mm}$. The clinical impression was Bowen's disease vs basal cell carcinoma. The lesion was curetted followed by cautery.

Results: The histological examination of routine H\&E sections revealed horizontal proliferation of non-pigmented atypical cells in the basal layer of the epidermis with focal pagetoid infiltration of the suprabasal epidermis. The solar elastotic dermis showed sheets of markedly atypical round, oval epithelioid and spindle shaped cells with enlarged hyperchromatic nuclei and prominent nucleoli. Occasional mitotic figures were present.

Immunohistochemically the atypical cells in the basal layer were strongly positive for SOX10 and S100. However, the dermal cells were strongly positive for CD10, CD68 and CD163 and negative for SOX10, S100, CKBS and P63. Morphological features and immunohistochemistry were consistent with a collision tumour composed of lentigo maligna and AFX/PDS.

Conclusions: To the best of our knowledge the association between lentigo maligna and AFX/PDS has not been reported previously; however, we are aware of two reports of association 